CASE REPORT

\author{
G. Zuccoli \\ I. Cravo \\ A. Bailey
}

A. Venturi

R. Nardone

\title{
Basal Ganglia Involvement in Wernicke Encephalopathy: Report of 2 Cases
}

SUMMARY: We present the neuroimaging and clinical findings in 2 nonalcoholic adult patients with WE as assessed by MR imaging. The first patient presented with gait ataxia and changes in consciousness. MR imaging disclosed bilateral lesions in the dorsal striatum and cerebellum. None of the regions typically affected in WE were involved. The second patient showed symmetric lesions in the posterior putamen associated with the alterations frequently and infrequently found WE.

ABBREVIATION: WE $=$ Wernicke encephalopathy

$\mathbf{W}$ ernicke encephalopathy is an uncommon neurologic disease resulting from dietary thiamine (vitamin $B_{1}$ ) deficiency. WE is characterized by changes in consciousness, ocular dysfunction, and ataxia. ${ }^{1-3}$ Previously, basal ganglia involvement, as demonstrated by MR imaging, was thought to differentiate the pediatric form of WE from the adult cases. ${ }^{3}$ Here, we present 2 adults with WE in whom involvement of the basal ganglia was observed.

\section{Case Reports}

\section{Case 1}

A 65-year-old malnourished woman with a clinical history of gastric cancer and gastrectomy was admitted to our institution. The patient was found to be in a stuporous state, but she was reactive to pain stimuli. Ocular examination disclosed decreased pupillary light reflexes, but her oculocephalic reflexes were normal. The Babinski sign was negative on both sides. Moderate hypotension (90/60 mm Hg) and tachycardia $(130 \mathrm{bpm})$ regressed after parenteral fluid infusion. Blood work-up showed moderate anemia (hemoglobin $=7.2 \mathrm{~g} / \mathrm{dL}$ ) and moderate hypokaliemia $\left(\mathrm{K}^{+}=3 \mathrm{mEq} / \mathrm{L}\right)$. Natremia values were within normal limits. MR imaging disclosed bilateral signal-intensity alteration involving the cerebellum and the dorsal striatum (Fig 1).

Findings in the remainder of the brain were unremarkable. CSF evaluation disclosed normal macroscopic, microscopic, and chemical findings. Routine analysis for protein 14-3-3 was positive, but findings of a genetic study of the human prion protein gene were negative. In WE, protein 14-3-3 may be detected. ${ }^{4}$ Results of tests for JC virus, herpes virus, enterovirus, cytomegalovirus, Toxoplasma gondii, listeria meningitis, mycobacterium tuberculosis, and borrelia were negative. Intravenous thiamine $900 \mathrm{mg} /$ day was given starting from day 2 after the admission. On day 9 , the patient was fully alert. However, gait ataxia and bilateral horizontal nystagmus were noted. On day 14, MR imaging follow-up disclosed significant decrease of the gait ataxia

Received April 26, 2010; accepted April 29.

From the Department of Radiology (G.Z.), University of Pittsburgh Medical Center, Children's Hospital of Pittsburgh, Pittsburg, Pennsylvania; Unidade Clinica de Neurorradiologia Serviço de Imagiologia (I.C.), Hospital Fernando Fonseca, Lisbon, Portugal; Department of Radiology (A.B.), University of Pittsburgh Medical Center, Pittsburgh, Pennsylvania; Departments of Radiology (A.V.) and Neurology (R.N.), "Franz Tappeiner" Hospital, Merano, Italy; and Department of Neurology and Neuroscience Insitute (R.N.), Christian Doppler Clinic, Paracelsus Private Medical University, Salzburg, Austria.

Please address correspondence to Giulio Zuccoli, MD, Department of Pediatric Radiology, University of Pittsburgh Medical Center, Children's Hospital of Pittsburgh, One Children's Hospital Dr, 4401 Penn Ave, Pittsburgh, PA 15224; e-mail: giulio.zuccoli@gmail.com DOI 10.3174/ajnr.A2185 and horizontal nystagmus. Three-month follow-up showed focal gliosis in the right putamen and extensive cerebellar atrophy.

\section{Case 2}

A 39-year-old woman with a history of a neoplasm of the uterus was hospitalized for radiation therapy-related jejunal perforation and intestinal occlusion. Emergency abdominal surgery with enterectomy and left colostomy was performed. One week after surgery, the patient developed nausea, vomiting, diplopia, horizontal nystagmus, and seizures. Laboratory test findings showed anemia (hemoglobin, 8.8 $\mathrm{g} / \mathrm{dL}$ ), but all other findings were unremarkable. MR imaging revealed typical and atypical WE findings, including the involvement of the putamen (Fig 2). On the basis of the clinical history, neurologic presentation, and MR imaging findings, a diagnosis of WE was made. Intravenous vitamin $B_{1}, 1000 \mathrm{mg} /$ day, was promptly administered. Twelve days following surgery, the patient developed sepsis. The patient died within a week of the onset of sepsis due to a gram-negative blood infection.

\section{Discussion}

Neuroimaging findings in WE typically show symmetric signal-intensity alterations in the mammillary bodies, medial thalami, tectal plate, and periaqueductal area. ${ }^{1-4}$ Selective involvement of the putamen has been reported only in the pediatric population and is considered an indicator of poor prognosis. ${ }^{3}$ In our patients, bilateral lesions in the putamen were observed. Patient 1 presented with signal-intensity alterations involving the dorsal striatum and the cerebellum. However, none of the typical lesions usually associated with WE were detected in patient 1 . To our knowledge, this is the first report in the literature demonstrating that unusual neuroimaging patterns of WE may be observed in the absence of typical findings. ${ }^{1-3}$ Although cerebellar involvement as observed in patient 1 has rarely been described in the neuroradiologic literature in association with $\mathrm{WE},{ }^{1,2}$ the cerebellum is highly dependent on thiamine metabolism, as evidenced by gait ataxia and neuronal loss. ${ }^{5}$ In patient 2 , the involvement of the putamen was associated with typical and atypical patterns of WE. Differential considerations of symmetric basal ganglia lesions in adults include various types of viral encephalitis. ${ }^{6}$ The presence of infectious disease - related symptoms is associated with these pathologic entities, and the hallmark combination of periaqueductal, tectal plate, and mammillary body involvement of WE is not present. ${ }^{1,2,6}$ Osmotic demyelination syndrome, variant Creutzfeldt-Jakob disease, methanol and carbon monoxide poisoning, multiple system atrophy, and 

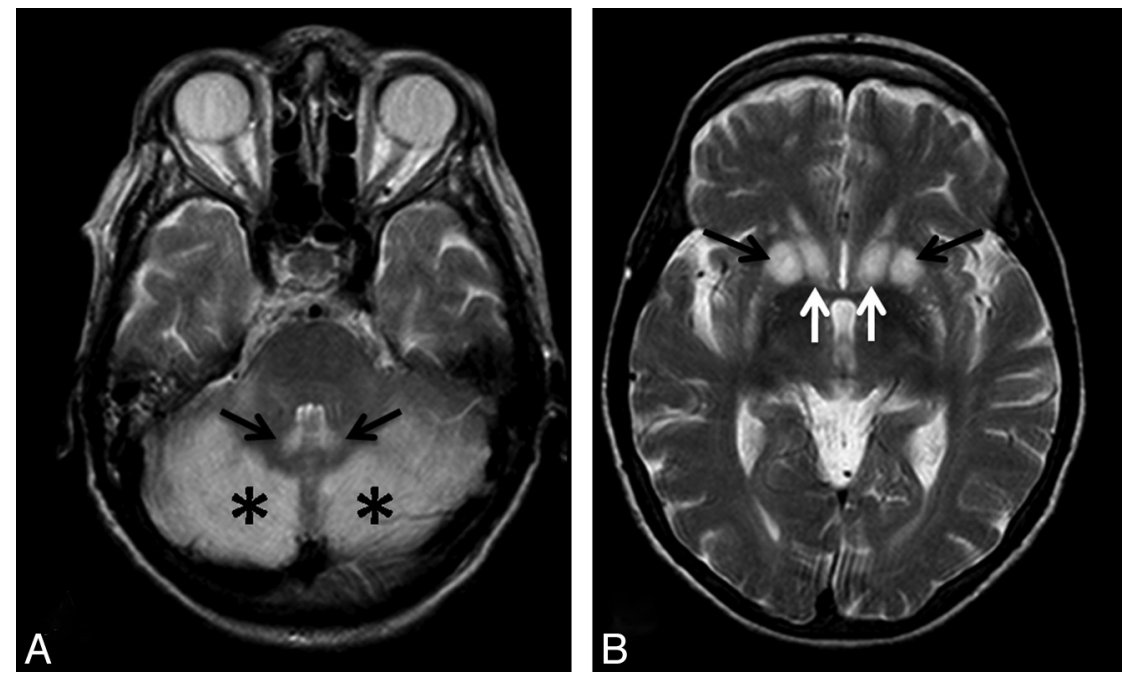

Fig 1. A, The cerebellar hemispheres (asterisks) and dentate nuclei (arrows) show T2 prolongation. B, The dorsal striatum (putamen, black arrows; caudate nucleus, white arrows) shows bilateral hyperintense alterations.
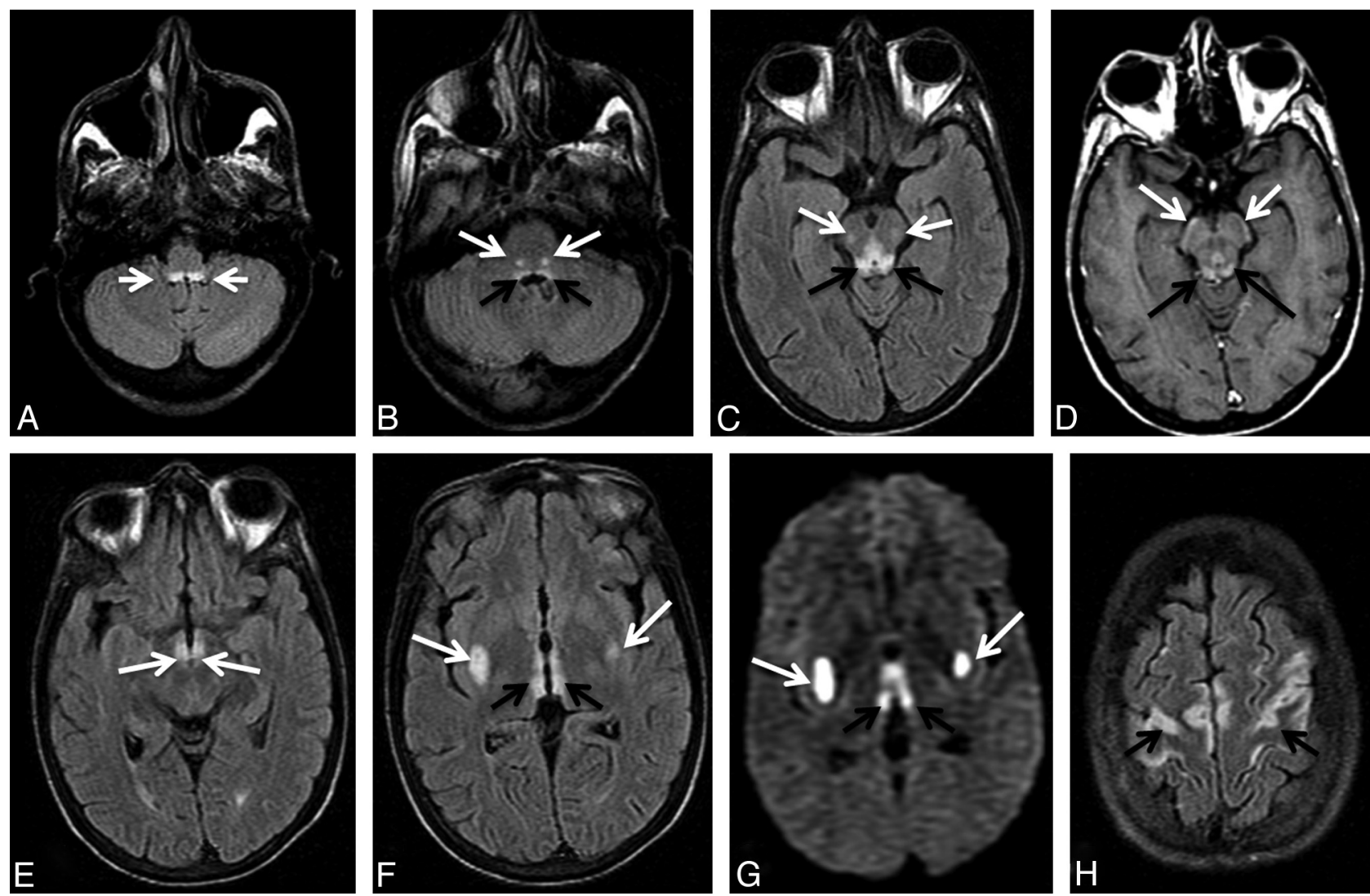

Fig 2. $A$, The prepositus hypoglossi nuclei regions show symmetric high signal-intensity alterations (arrows). $B$, The abducens nuclei regions show symmetric hyperintense alterations (black

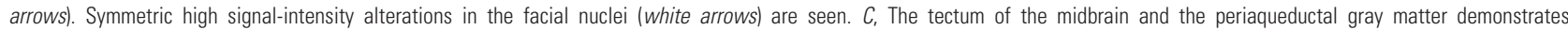
signal-intensity alterations (black arrows). Focal lesions in the medial lemniscus are seen bilaterally (white arrows). $D$, The inferior quadrigeminal plate, the periaqueductal gray matter (black arrows), and the corticospinal tracts (white arrows) show contrast enhancement. E, Signal-intensity alterations of the mamillary bodies are detected (white arrows). F and G, Symmetric alterations of the posterior putamen (white arrows) and periventricular region of the third ventricle (black arrows) are seen on fluid-attenuated inversion recovery and diffusion-weighted images, respectively. $H$, Signal-intensity alterations are seen in the motor strip (arrows).

hypoxic injury represent other possible differential diagnoses of bilateral basal ganglia signal-intensity alteration. ${ }^{6}$

Symmetric involvement of the cranial nerve nuclei in patient 2 is interesting because this is an atypical finding recently found to be characteristic of nonalcoholic WE. ${ }^{1-3}$ Furthermore, medial lemniscus and corticospinal tract involvement has not been previously described, thus supporting the hypothesis that WE selectively affects not only the gray matter nuclei but also the white matter tracts at the brain stem level. Patient 2 also showed involvement of the cortex, consistent with previous reports that have demonstrated an association of this area with nonalcoholic WE. ${ }^{1,2}$ 


\section{Conclusions}

In patients presenting with a history of malnutrition, alcoholism, or malignancy associated with basal ganglia lesions, differential diagnosis should include WE, even if none of the typically affected regions are involved on MR imaging. Previously it was hypothesized that basal ganglia involvement, specifically at the level of the putamen, was due to an increase in the rate of thiamine-dependent metabolism in the pediatric population. ${ }^{3}$ However, it now appears that WE may affect the basal ganglia in adults as well as children, even if this finding may be considered exceedingly rare.

\section{References}

1. Zuccoli G, Santa Cruz D, Bertolini M, et al. MR imaging findings in 56 patients with Wernicke encephalopathy: nonalcoholics may differ from alcoholics. AJNR Am J Neuroradiol 2009;30:171-76

2. Zuccoli G, Pipitone N. Neuroimaging findings in acute Wernicke's encephalopathy: review of the literature. AJR Am J Roentgenol 2009;192: 501-08

3. Zuccoli G, Siddiqui N, Bailey A, et al. Neuroimaging findings in pediatric Wernicke encephalopathy: a review. Neuroradiology 2010;52:523-39. Epub 2009 Oct 21

4. Bertrand A, Brandel JP, Grignon Y, et al. Wernicke encephalopathy and Creutzfeldt-Jakob disease. J Neurol 2009;256:904-09

5. Mulhulland PJ. Susceptibility of the cerebellum to thiamine deficiency. Cerebellum 2006;5:55-63

6. Brami-Zylberberg F, Méary E, Oppenheim C, et al. Abnormalities of the basal ganglia and thalami in adults. J Radiol 2005;86:281-93 